\title{
A Modified Fixed-Newton's Method Via Mid-Point Approach for Nonlinear Systems of Equations
}

\author{
H. A. Aisha, K. Halima and M.Y Waziri \\ Department of Mathematics, Faculty of Science, Bayero University Kano, Kano, Nigeria
}

\begin{abstract}
The major shortcomings of Classical Newton's method for nonlinear equations entail computation of Jacobian matrix and solving systems of $n$ linear equations in every iteration. Mostly function derivatives are quit costly and Jacobian is computationally expensive which requires storage of matrix in each iteration. The appealing approach is based on Fixed Newton's but the method mostly requires high number of iteration as the dimension of the systems increases due to less Jacobian information in every iteration. In this paper, we introduce a new procedure via two-step scheme that will reduce the well known shortcomings of Fixed and classical Newton methods. Numerical experiments are carried out which shows that, the proposed method is very encouraging are presented.
\end{abstract}

Keywords: Nonlinear equations, Equations, Fixed Newton's, Inverse Jacobian.

Let us consider the problem of finding the solution of nonlinear equations

$F(x)=0$,

Where $F: R^{n} \rightarrow R^{n}$ s a nonlinear mapping. Often, the mapping, $\mathrm{F}$ is assumed to satisfying the following assumptions:

A1. F is continuously differentiable in a open neighborhood $S$ of a solution $x^{*} \in S$ of the system (1.1),

A2. There exists a solution $x^{*}$ where $F\left(x^{*}\right)=0$

A3.

$F^{\prime}\left(x^{*}\right) \neq 0$ is invertible.

The well known method for finding the solution to (1), is the classical Newton's method which generates a sequence of iterates $\left\{x_{k}\right\}$ from a given initial point $\mathrm{x} 0$ via

$x_{k+1}=x_{k}-F^{\prime}\left(x_{k}\right)^{-1} F\left(x_{k}\right), \quad \mathrm{k}=0,1,2 \ldots$,

where $F^{\prime}\left(x_{k}\right)$ is the Jacobian of $\mathrm{F}$. The attractive features of this method are; rapid convergence and easy to implement. Nevertheless, it requires the computation of the Jacobian matrix which entails the first-order derivatives of the systems. The computational budget of Newton's method mostly becomes more expensive, particularly as the dimension of the nonlinear systems increases due to computation and storage of Jacobian matrix in every iteration. In practice some derivatives are highly costly to obtain or cannot be done precisely, in this case Newton's method could not be a good candidate [1, 3, 4]. Many efforts have been made, by a substantial number of researchers to overcome the well know disadvantage of Newton's method[8]. The simplified and easiest variant of Newton method is Fixed Newton's method. This method saves a lot of computational burdens of the Jacobian matrix $F^{\prime}\left(x_{k}\right)$, by approximating the Jacobian with the Jacobian at $x_{0}$ (Initial guess) i.e

$F^{\prime}\left(x_{k}\right) \square F\left(x_{0}\right)$

for all $k$.

The Fixed Newton's method generates an iterative sequence $\left\{x_{k}\right\}$ via the following algorithm:

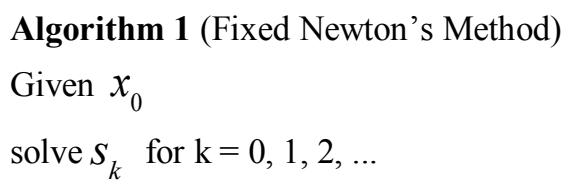


$F^{\prime}\left(x_{0}\right) s_{k}=-F\left(x_{k}\right)$

Update

$x_{k+1}=x_{k}+s_{k}$

Despite its good quality, Newton's chord method mostly requires more number of iteration and the convergence may even be lost because of less Jacobian information in each iteration $[2,6,7]$. In fact, most of the variants of fixed method do not work

2perfectly. In this paper we present a simple modification of fixed Newton's method for nonlinear systems, by using mid-point stratergy. The main idea behind this task is that, we aim at reducing the number of iteration and to correct the convergence property of Fixed Newton's method. Our is significantly cheaper than classical Newton's method and faster than Fixed Newton's method with respect to CPU time in general. We organize the paper as follows: Section 2 presents the new variant of fixed Newton's method. Some numerical results and discussion are given in section 3 , and finally conclusion is reported

\section{Derivation Process}

In this section, we shall present our new modification of Fixed Newton's method (MFNM). The proposed method generates a sequence of points

$x_{k+1}=x_{k}-F^{\prime}\left(x_{0}\right)^{-1} F\left(x_{k}\right)$,

for $\mathrm{k}=0,1,2, \ldots$

It is vital to mention that, [5] have reported that, the undesirable performance behaviors of Newton's chord methodespecially when solving high dimensional systems of nonlinear equations is associated with the insufficient Jacobian information in each iteration. The validation associated to our procedure is to enhance the convergence properties as well as improving numerical stability. This is made possible by employing Mid- Point strategy on the iterates. With this scheme, we expect our method to yields a significant reduction in CPU time consumption and number of iteration compared to Fixed Newton's method.

\section{Algorithm 2 (Modified Fixed Newton's method(MFNM)))}

Step I: Given $\mathrm{x}_{0}$, Eand set $\mathrm{k}=0$.

Step II: Compute $\mathrm{J}\left(\mathrm{x}_{0}\right)^{-1}$

Step III: Compute $\mathrm{F}\left(\mathrm{x}_{\mathrm{k}}\right)$

Step IV: Check stopping condition.,i.e $\left\|F\left(x_{k}\right)\right\| \leq 10^{-3}$, If yes stop, else go to Step

Step V: Compute $\alpha_{k}=x_{k}-J\left(x_{0}\right)^{-1} F\left(x_{k}\right)$,

Step VI: Compute $q_{k}=\frac{\alpha_{k}+x_{k}}{2}$

Step VII: Compute $\mathrm{F}\left(\mathrm{q}_{\mathrm{k}}\right)$

Step VIII: Set $x_{k+1}=q_{k}-J\left(x_{0}\right)^{-1} F\left(q_{k}\right)$,

Step IX: Set $\mathrm{k}=\mathrm{k}+1$ and go back to step II.

\section{Numerical results}

This section presents the performance of MFNM method, when compared with Fixed Newton method (FNM) and Newton's method (CN). The codes are written in MATLAB 7.4 with a double precision computer, the stopping condition used is:

$\left\|F\left(x_{k}\right)\right\| \leq 10^{-3}$

Four (5) benchmark problems are considered. We further design the codes to terminate whenever one of the following happens;

(i) The number of iteration is at least 600 but no point of $x_{k}$ that satisfies (4) is obtained;

(ii) (ii) Insufficient memory to initial the run.

In the following, some details on the benchmarks test problems are presented: 


\section{Problem 1 System of nonlinear equations:}

$$
\mathrm{F}_{2}=\mathrm{x}_{1}^{4}+5 \mathrm{x}_{2}-6
$$

$$
\mathrm{F}_{1}=10 \mathrm{x}_{1}^{2}+\sin \left(\mathrm{x}_{2}\right)-20
$$

$$
\mathrm{x}_{0}=(1, \quad 1)
$$

Problem 2 System of nonlinear equations:

$$
\begin{array}{cc}
\mathrm{F}_{2}=\mathrm{x}_{2}^{2}-1 & \mathrm{~F}_{1}=\mathrm{x}_{1}^{2}-1 \\
& \mathrm{x}_{0}=(0.1,
\end{array}
$$

Problem 3 System of nonlinear equations:

$\mathrm{F}_{1}=\exp \left(\mathrm{x}_{1}\right)+\mathrm{x}_{2}-1$

$\mathrm{F}_{2}=\exp \left(\mathrm{x}_{2}\right)+\mathrm{x}_{1}-1$

$\mathrm{x}_{0}=(-0.5,-0.5)$,

Problem 4 System of nonlinear equations:

$$
\mathrm{F}_{1}=\mathrm{x}_{1}^{3}+\mathrm{x}_{1}^{2}
$$

$\mathrm{x}_{0}=(0.8,0.8)$,

$$
\mathrm{F}_{2}=\mathrm{x}_{1}^{2}-\mathrm{x}_{2}^{2}
$$

$$
\begin{aligned}
& \text { Problem 5 System of nonlinear equations: } \\
& \mathrm{F}_{1}=\exp \left(\mathrm{x}_{1}\right)-1 \\
& \mathrm{~F}_{2}=\exp \left(\mathrm{x}_{2}\right)-1 \\
& \mathrm{x}_{0}=(-0.5,-0.5)
\end{aligned}
$$

\begin{tabular}{|c|c|c|c|c|}
\hline Problem & $\mathrm{x}_{0}$ & NM & FNM & MFNM \\
\hline 1 & $(1,1)$ & 6 & 9 & 5 \\
\hline 2 & $(0.1,0.1)$ & 2 & 5 & 3 \\
\hline 3 & $(-0.5,-0.5)$ & 3 & 5 & 4 \\
\hline 4 & $(0.8,0.8)$ & 8 & - & 3 \\
\hline 5 & $(-0.5,-0.5)$ & 3 & 17 & 3 \\
\hline
\end{tabular}

The numerical results presented in Table 1 demonstrate clearly the proposed method(MFNM) shows a good improvement, when compared with NM and FNM respectively. In addi- tion, it is worth mentioning, the MFNM method does not require more storage locations than classic Newton's and Fixed Newton's methods Respectively. Moreso, the proposed method (MFNM) is faster than FNM methods and required little time to solve the problems when compared to the other Newton-like methods.

\section{Conclusion}

We have suggested a Modified Fixed Newton's method for solving nonlinear systems of equations. The method uses Mid- Point approach and the anticipation has been to further improve the performance of Fixed newton's method for handling nonlinear sysytems of equations. It is also worth mentioning that the MFNM method is capable of significantly reducing the execution time ( CPU time) and Number of iteration, as compared to NM and FNM methods while maintaining good accuracy of the numerical solution to some extend Numerical experiment presented, shows that in all the tested problems, MFNM Method is very promising . Finally, we can claim that, our approach is a good candidate for solving systems of nonlinear equations.

\section{References}

[1]. M.Y. Waziri and Z. A. Majid, 2012A new approach for solving dual Fuzzy nonlinear equations, Advances in Fuzzy Systems. Volume 2012, Article ID 682087, 5 pages doi:10.1155/2012/682087 no. 25, 1205 - 1217.

[2]. Dennis, J, E., 1983, Numerical methods for unconstrained optimization and nonlinear equations, Prince-Hall, Inc., Englewood Cliffs, New Jersey

[3]. C.T. Kelley Iterative Methods for Linear and Nonlinear Equations", SIAM, Philadelphia,

[4]. M. Y. Waziri, W.J. Leong, M. A. Hassan,M. Monsi, A New Newton method with diagonal Jacobian approximation for systems of Non-Linear equations. Journal of Mathematics and Statistics Science Publication.6 :(3) 246-252 (2010).

[5]. M.Y. Waziri, W.J. Leong, M.A. Hassan, M. Monsi, Jacobian computation-free New- ton method for systems of Non-Linear equations. Journal of numerical Mathematics and stochastic. (2010); $2: 1: 54-63$.

[6]. Leong, W. J., Hassan, M. A and Waziri, M. Y., 2011, A matrix-free quasi- Newton method for solving large-scale nonlinear systems, Comput. Math. App. 62 5: 2354- 2363.

[7]. Byeong, C. S. Darvishi, M. T. and Chang, H. K. 2010. A comparison of the New- tonKrylov method with high order Newton-like methods to solve nonlinear systems . Appl. Math. Comput. 217: 3190-3198.

[8]. Hao. L. and Qin., N., Incomplete Jacobian Newton method for nonlinear equation, Computers and Mathematics with Applications $56,218-227(2008)$. 